\title{
Retrograde Oxygen Persufflation of Kidney: Experiment on an Animal
}

\author{
Moláček $\mathbf{J}^{1,2 *}$, Třeška $V^{1,2}$, Opatrný $V^{1}$, Matějka $R^{3}$ and Baxa $\mathrm{J}^{2}$
}

${ }^{1}$ Department of Surgery, University Hospital in Pilsen, Czech Republic

${ }^{2}$ Faculty of Medicine in Pilsen, Charles University in Prague, Czech Republic

${ }^{3}$ Czech Technical University in Prague, Faculty of Biomedical Engineering in Kladno, Czech Republic

\begin{abstract}
Objective: There is still a lack of organs for transplantation purposes. In the field of kidney and liver transplantation one of available solutions is the use of organs from so-called marginal donors. These donors can be e.g. non-heartbeating donors (NHBD). In these cases perfusion and preservation of organs intended for transplantation is generally more difficult. Retrograde oxygen persufflation (ROP) may be one of the possible solutions of this issue. This method is based on retrograde perfusion by oxygen through the renal vein thus reconditioning the organ.
\end{abstract}

Materials and methods: We have operated on 10 animals (porcine models). In all animals was simulated ischemic injury of right kidney. In Group $A(\mathrm{~N}=5)$ were kidneys after explantation perfused with retrograde oxygen persufflation. In group $B(N=5)$ were kidneys perfused intrarterialy like usually in clinical practice. After perfusion were all kidneys again transplanted to the same animal. Quality of graft restitution was evaluated by urea level taken from renal vein and by histopathological analysis after explantation.

Results: We have found no statistically significant differences between the groups $A$ and $B$ in urea levels after transplantation as wel as we have found no significant differences in quality of kidney parenchyma restoration in both groups.

Conclusions: Retrograde oxygen persufflation is able to protect and restore kidney parenchyma.

Keywords: Kidney transplantation; Retrograde oxygen persufflation; Organ preservation

\section{Introduction}

Today, we still see a lack of organs for transplantation purposes. In the field of kidney and liver transplantation one of available solutions is the use of organs from so-called marginal donors. These donors can be e.g. non-heart-beating donors (NHBD). In these cases perfusion and preservation of organs intended for transplantation is generally more difficult. In the current clinical practice, intraarterial renal perfusion is applied at first in situ. The graft is later, after explanation, perfused using a perfusion pump. An alternative is the customary storage in an ice-cold solution ("cold storage") applied after in situ perfusion. Kidneys taken from non-heart-beating donors are always injured by warm ischemia. A method, which would improve the quality of organs, is still being looked for. Retrograde oxygen persufflation (ROP) may be one of the possible solutions of this issue. This method is based on retrograde perfusion by oxygen through the renal vein. Our objective was to simulate warm ischemia in a porcine kidney and then to preserve the graft only using venous retrograde oxygen persufflation. After the kidney retransplantation, we evaluated the graft function, the degree of its injury and compared such modalities with grafts subjected to standard intraarterial perfusion by a preservation solution. The goal was to find an answer to the question whether the ROP could be beneficial for the preservation and recondition of organs injured by warm ischemia.

\section{Methodology}

The animal used for the experiment was a (male) porcine animal with the weight of approx. $30 \mathrm{~kg}$. The operations were performed at the Experimental Medicine Centre of the Faculty of Medicine in Pilsen. The animals were provided with care pursuant to legislation of the Czech Republic applicable to work with laboratory animals and of course this experiment followed EU regulation for research on laboratory animal. The project was approved by Ethical Committee of School of Medicine in Pilsen Charles University in Prague.

The design of the experiment: In group $\mathbf{A}$ ( $\mathrm{N}=5$ animals) the experimental animal was put into general anaesthesia (Thiopental, Calypsol, Fentanyl). We reached retroperitoneum throughout medial laparotomy, exposed the renal vessels in the right kidney. A kidney pedicle clamp was applied for 20 minutes (simulation of warm ischemia). Then the explantation of the kidney was performed and at the same time the first renal biopsy sample (biopsy - IA) was taken. The bioptic sample was taken in the form of a wedge excision of renal parenchyma including cortex as well as medulla. After explantation, the renal graft was connected to an insufflator by which the graft was perfused in a retrograde manner with moisturized gaseous oxygen under the pressure of $18 \mathrm{~mm}$ of mercury. The perfusion cannula was applied into the renal vein. During the procedure the graft is placed in a cold perfusion solution (Custodiol) at the temperature of $+4{ }^{\circ} \mathrm{C}$ (for a detailed description, see below) (Figure 1). It was necessary to needlepuncture several perforations into the renal parenchyma to allow gaseous oxygen to release. The perfusion lasted 60 minutes. At the end of this interval, the second renal biopsy sample was taken (biopsy - IIA). Then the renal graft was retransplanted to the same porcine animal and the other-sided (left-sided) nephrectomy was performed. During the transplantation, the renal artery was anastomosed to the aorta (end-toside anastomosis) and the renal vein to the inferior vena cava (end-toside anastomosis). After the restoration of blood circulation through the graft, a sample of venous blood was taken from the renal vein of the transplanted kidney every 30 minutes to determine the renal functions markers. After 120 minutes the graft was explanted, the third bioptic

*Corresponding author: Jiri Molacek M.D., Ph.D. Department of Surgery, University Hospital in Pilsen Czech Republic, Tel: 42037710 4275; E-mail: molacek@fnplzen.cz

Received June 22, 2015; Accepted July 10, 2015; Published July 19, 2015

Citation: Moláček J, Třeška V, Opatrný V, Matějka R, Baxa J (2015) Retrograde Oxygen Persufflation of Kidney: Experiment on an Animal. J Transplant Technol Res 5: 147. doi:10.4172/2161-0991.1000147

Copyright: @ 2015 Moláček J, et al. This is an open-access article distributed under the terms of the Creative Commons Attribution License, which permits unrestricted use, distribution, and reproduction in any medium, provided the original author and source are credited. 


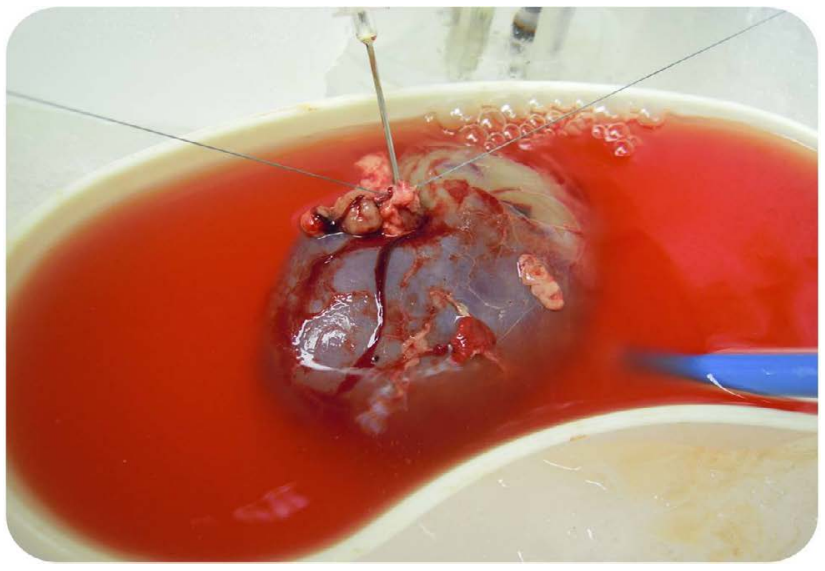

Figure 1: Kidney retrograde oxygen persufflation

sample of the kidney (biopsy - IIIA) was taken and the experimental animal put to death by a cardioplegic solution

In group $\mathbf{B}(\mathrm{N}=5$ animals), renal ischemia was also simulated for 20 minutes by the kidney pedicle clamping. After nephrectomy, the renal graft was routinely perfused using the Custodiol perfusion solution under the stationary hydrostatic pressure at the temperature of $+4{ }^{\circ} \mathrm{C}$. The cannula was applied customarily into the renal artery (Figure 2). The perfusion was applied for the same period as in the group A - 60 minutes. Then the kidney was retransplanted in the same manner as was the other-sided nephrectomy. Blood samples were taken again from the renal vein. The bioptic samples were taken at identical intervals (biopsy IB - IIIB). After 120 minutes the animals were put to death identically as in the group A (Figure 3 ).

The histopathological examination of the graft was performed by a qualified pathologist who generally assessed the degree of injury to the nephron (based on the scale provided hereunder). The items assessed were the quality of the glomerulus perfusion, the presence of microthrombi and, where applicable, the presence of acute tubular necrosis.

Level 1... Complete perfusion, no erythrocytes in capillary vessels

Level 2... Incomplete perfusion, occasional groups of erythrocytes in capillary vessels (in particular in the glomerulus)

Level 3... The kidney remained non-perfused (physiological condition)

Level 4... Non-perfused kidney, the presence of acute tubular necrosis

The study was blinded for the pathologist.

In this phase of the experiment, a level of urea in blood serum was evaluated from the vein blood samples.

\section{Description of the ROP procedure performance}

Experimental unit for the mechanical perfusion parameters monitoring and control: For the needs of the monitoring of parameters during the experiments a multi-functional platform based experimental unit using the field-programmable gate array (FPGA) technology was designed. Specifically, the NI-PCI-7831R card (National Instruments, Austin, TX, USA) installed in an IPC was concerned. The individual sensors measuring the process quantities (pressure, flow rate, temperature) were connected to this card together with the electronic system for the control of the pumps to provide forced mechanical perfusion. Pressure was measured both by sensors MPXV5010 and MPXV4002 (Freescale Semi., Austin,TX, USA) and pressure liquid chambers TruWave (Edwards Lifesciences, Irvine, CA, USA). Temperature measuring was performed by standard temperature sensors Pt-100 placed into glass cases (Theta90, Prague, Czech Republic) and sensors for surface temperature measuring with NTC 30k@25 ${ }^{\circ} \mathrm{C}$ (LHL s.r.o., Ústí n. Labem, Czech Republic). Flow rate was measured using the inline flow rate sensor Sonoflow IL.52 (Sonotec, Halle, Germany). The sensors were completed with the necessary electronic system to ascertain the required accuracy and stability. The forced perfusion was induced using gear pumps (Diener, Embrach, Switzerland) with the electronic performance control system based on pulse width modulation. Due to this control, it was possible to adjust the flow rate parameters infinitely, including the simulation of the pulsatile wave. For the needs of the experimental unit control, parameters monitoring and log creating, a special-purpose software application was developed.

ROP: During the retrograde oxygen perfusion, the pressure of oxygen was set at $18 \mathrm{~mm} \mathrm{Hg}(2.4 \mathrm{kPa})$ with a deviation of $+/-0.2 \mathrm{~mm}$ $\mathrm{Hg}$ (this data is based on a reference). Pressure was measured in the tee-branch between the catheter inserted in the supply tube using the MPXV5010 pressure sensor. The temperature of the dialysate, in which the kidney was tempered, was $4^{\circ} \mathrm{C}$ maintained by the laboratory temperature controller equipped with a cooling system.

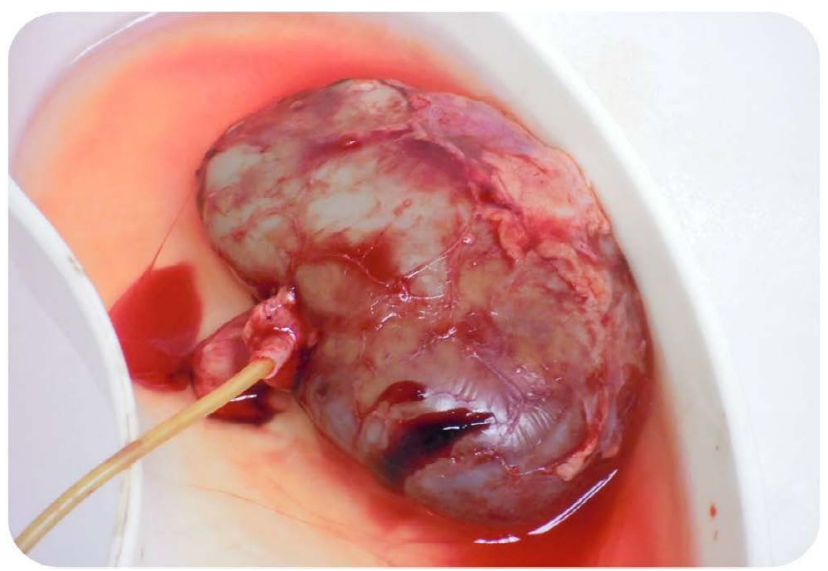

Figure 2: Intraarterial perfusion.

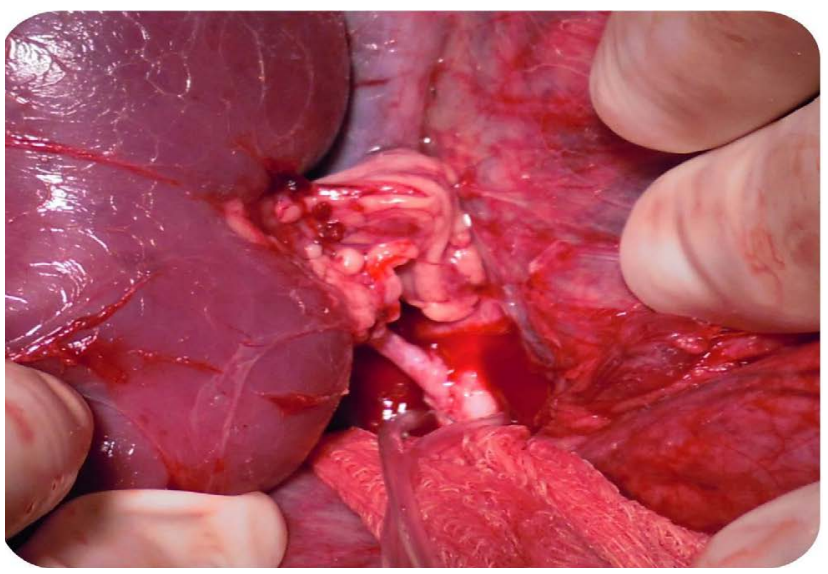

Figure 3: Condition after the graft retransplantation. 
Intraarterial perfusion: In the case of the grafts subjected to intraarterial perfusion by the defined hydrostatic pressure also the flow rate through the kidney and the pressure, which was measured in the second lumen of the supply catheter, were recorded. In this manner, the error of measurement caused by self-fluidic resistance was minimised. The liquid chamber TruWave was used as a sensor. The dialysate was cooled down to the temperature of $4^{\circ} \mathrm{C}$ using the laboratory temperature controller equipped with a cooling system but due to its recirculation and the initial heating by the organ, the temperature during the experiment was maintained within the range of $3.9-6.4^{\circ} \mathrm{C}$. The temperature of the organ dropped to the value of approx. $5.2^{\circ} \mathrm{C}$ measured on the surface.

\section{Results}

No technical problems with the graft perfusion or transplantation as such were found in any of the presented experimental animals. In two cases (one case in each group) an aggravated segmental perfusion was discovered upon the pole artery interruption.

\section{Evaluation of urea levels in blood serum}

Table 1 provides the values of urea in blood taken from the renal vein after the kidney retransplantation at individual intervals $(0,30,60$, 120 minutes).

The resulting value $\mathrm{p}$ of the Repeated Measure ANOVA test for differences between the groups $\mathrm{A}$ and $\mathrm{B}$ is $\mathrm{p}=0.843$, which means that no statistically significant differences were found between the groups $\mathrm{A}$ and B (Graph 1).

Table 1: Levels of urea in $\mathrm{mmol} / \mathrm{l}$ in individual animals at the times ranging from 0 to 120 minutes.

\begin{tabular}{|c|c|c|c|c|}
\hline $\begin{array}{c}\text { Animal number } \\
\text { (group) }\end{array}$ & $\begin{array}{c}\text { Urea }(\mathrm{mmol} / \mathrm{l}) \\
0 \mathrm{~min}\end{array}$ & $\begin{array}{c}\text { Urea }(\mathrm{mmol} / \mathrm{l}) \\
30 \mathrm{~min}\end{array}$ & $\begin{array}{c}\text { Urea }(\mathrm{mmol} / \mathrm{l}) \\
60 \mathrm{~min}\end{array}$ & $\begin{array}{l}\text { Urea }(\mathrm{mmol} / \mathrm{l}) \\
120 \mathrm{~min}\end{array}$ \\
\hline $1(\mathrm{~A})$ & 4 & 4 & 6 & 7 \\
\hline $2(\mathrm{~A})$ & 5 & 6 & 6 & 7 \\
\hline $3(\mathrm{~A})$ & 8 & 8 & 8 & 10 \\
\hline $4(\mathrm{~A})$ & 8 & 7 & 9 & 9 \\
\hline $5(\mathrm{~A})$ & 6 & 7 & 8 & 8 \\
\hline $6(\mathrm{~B})$ & 5 & 5 & 7 & 7 \\
\hline 7 (B) & 7 & 7 & 8 & 9 \\
\hline 8 (B) & 4 & 6 & 12 & 8 \\
\hline 9 (B) & 7 & 6 & 8 & 8 \\
\hline 10 (B) & 5 & 5 & 7 & 7 \\
\hline
\end{tabular}

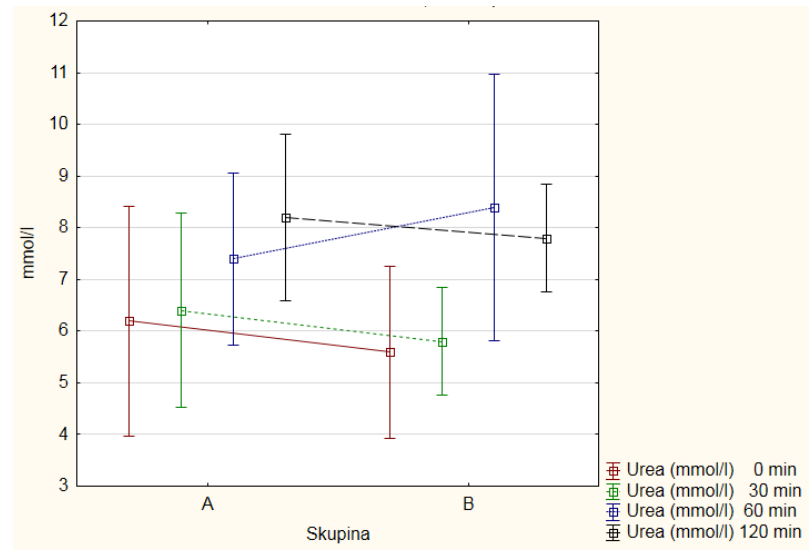

Graph 1: Levels of urea in $\mathrm{mmol} / \mathrm{l}$ in individual animals at the times ranging from 0 to 120 minutes.

\section{Histopathological evaluation of the bioptic samples}

Table 2 provides the level of the quality of the nephron perfusion plus the overall improvement of the parenchyma quality (the presence of microthrombi, acute tubular necrosis)

Scale 1 - the best to 4 - the worst

The value according to the Fisher's exact test for time I was $\mathrm{p}=1.00$, which means higher than 0.05 . The level of the kidney perfusion 1,2 , 3 or 4 thus does not differ much statistically between the group A and B (Graph 2).

The value according to the Fisher's exact test for time II was $\mathrm{p}=1.00$, which means higher than 0.05 . The level of the kidney perfusion 1,2 , 3 or 4 thus does not differ much statistically between the group A and B (Graph 3).

The value according to the Fisher's exact test for time III was $p=1.00$, which means higher than 0.05 . The level of the kidney perfusion 1,2 , 3 or 4 thus does not differ much statistically between the group A and B (Graph 4).

\section{Discussion}

A permanent lack of organs for transplantation purposes results in a higher tolerance of the acceptance of marginal donors. This topic is closely related to the implementation of new methods of the preservation of grafts and a so-called recondition of organs after explantation from the donor. This approach is justified by very good results in particular of renal grafts from NHBD achieved in clinical practice [1-3]. The primary factor injuring parenchyma is warm ischemia. Warm ischemia is found especially in (NHBD). The procedure of a renal graft preservation and recondition applied at individual clinics often differs [4-8].

The retrograde oxygen persufflation (ROP), which was discovered accidentally, was first described in the 1970s [9]. A number of experimental studies were performed in the past $[10,11]$. For the first time probably in clinical practice, the ROP was applied to kidneys by Rolles [12] in 1989. Studies comparing the quality of grafts preserved by the ROP with those routinely subjected to cold storage or intraarterial perfusion have been performed on a repetitive $[13,14]$. It has been proven that the ROP can be in many aspects even more beneficial than the customary preservation both for kidneys, and for liver. A majority of experiments concerned small-sized animals (rats, dogs). Other experiments with the ROP dealt in particular with the bioenergy condition of grafts and their function after transplantation [15]. More recent similar experiments were performed on porcine animals [16,17]. Recent paper by Suszynski in Cryobiology describes very well all preservation methods in different organs. In our experimental study, we wished to verify the suitability of the ROP for a large experimental animal. From this point of view, a porcine animal is an absolutely ideal bio-model. Due to both its anatomy, and the pathophysiology of the injury caused by warm ischemia.

The persufflator was designed specifically for this experimental project. Specific and fully controlled values of perfusion pressure and flow rate are provided. Technically, this concerns a very easy and not at all costly procedure. In this phase of the experiment, we opted to perform the evaluation of the graft's quality by the histopathological analysis of the graft (both after preservation itself, and after the subsequent transplantation). The own scale of graft quality (1 to 4) has been applied at our clinic on a long-term basis (in addition to the standard Remuzzi's score) for the evaluation of grafts from NHBDs. The other evaluation factor was the function of the transplanted kidney, 
Citation: Moláček J, Třeška V, Opatrný V, Matějka R, Baxa J (2015) Retrograde Oxygen Persufflation of Kidney: Experiment on an Animal. J Transplant Technol Res 5: 147. doi:10.4172/2161-0991.1000147

Page 4 of 5

Table 2: Level of quality of the renal graft perfusion.

\begin{tabular}{|c|c|c|c|}
\hline Animal (group) / Sample & I & II & III \\
\hline 1 (A) & 2 & 2 & 3 \\
\hline 2 (A) & 3 & 2 & 2 \\
\hline $3(A)$ & 1 & 2 & 1 \\
\hline $4(A)$ & 2 & 3 & 2 \\
\hline $5(A)$ & 2 & 2 & 3 \\
\hline $6(B)$ & 2 & 1 & 3 \\
\hline $7(B)$ & 3 & 2 & 2 \\
\hline $8(B)$ & 3 & 3 & 2 \\
\hline $9(B)$ & 1 & 1 & 2 \\
\hline $10(B)$ & 1 & 4 & 3 \\
\hline
\end{tabular}

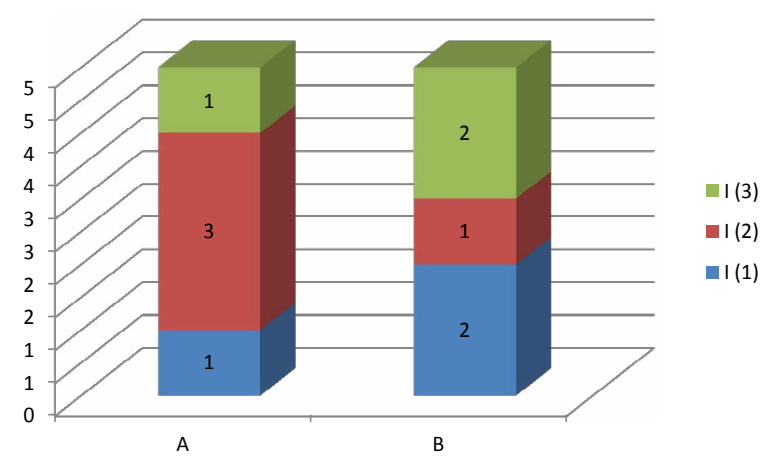

Graph 2: Expression of the quality of the kidney perfusion using the Fisher's exact test for time I.

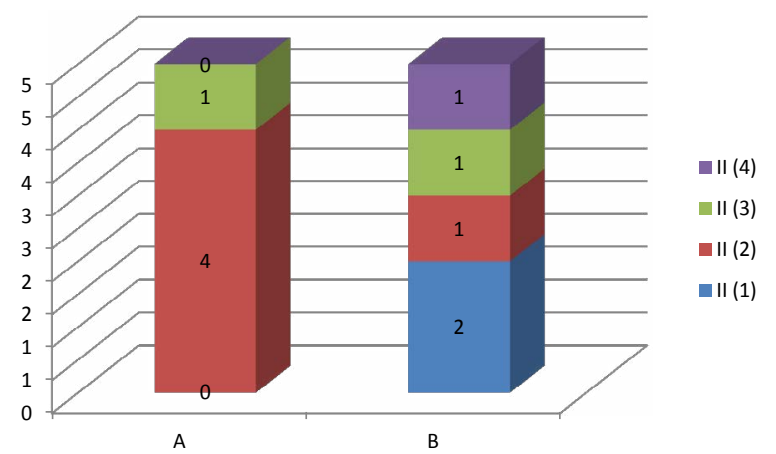

Graph 3: Expression of the quality of the kidney perfusion using the Fisher's exact test for time II.

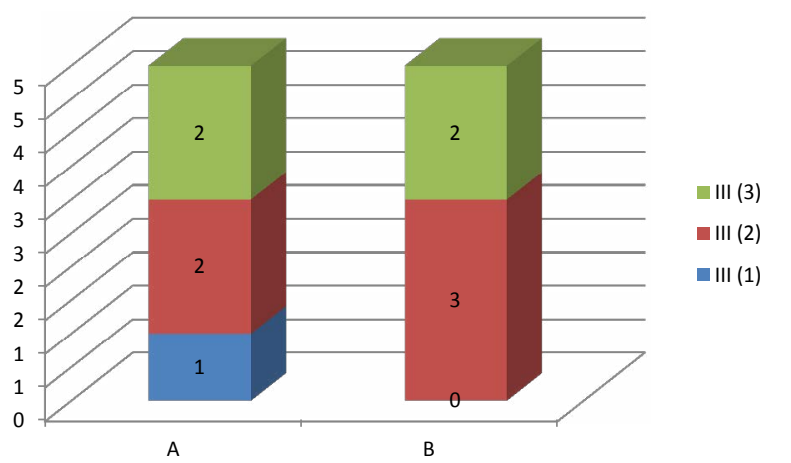

Graph 4: Expression of the quality of the kidney perfusion using the Fisher's exact test for time III. which was objectivised by regular sampling of vein blood from the graft. The marker of the graft function at this point was the level of urea (contralateral nephrectomy). We are aware of the fact that the view of ours is simplifying to a certain extent. However, our goal was to find out whether the ROP can be a certain alternative for the recondition of a renal graft.

Our results have proven the identical quality of grafts preserved by both standard intraarterial perfusion, and retrograde oxygen persufflation. We have discovered no significant differences both in the histopathological evaluation of the extracted grafts (tested using the Fisher's exact test in a contingency table for each of the times I, II and III independently), and in the values of urea in animals after the transplantation of the respective grafts. Our results are in accordance with references. We have not proven the opinion of some authors that the ROP may be even superior compared to standard perfusion or cold storage $[17,18]$. On the other hand, we have proven beyond reasonable doubt that a mere ROP is able to protect and restore parenchyma (Figures 4 and 5).

In the next phase of the experiment, we would like to extend both the period of warm ischemia of the graft, and the period of restitution using the ROP. In addition, we would like to extend the survival of the experimental animal at least in the order of days. It is also necessary to use new laboratory markers of the renal graft function. A combination of the ROP with the pharmacological application of free oxygen radicals

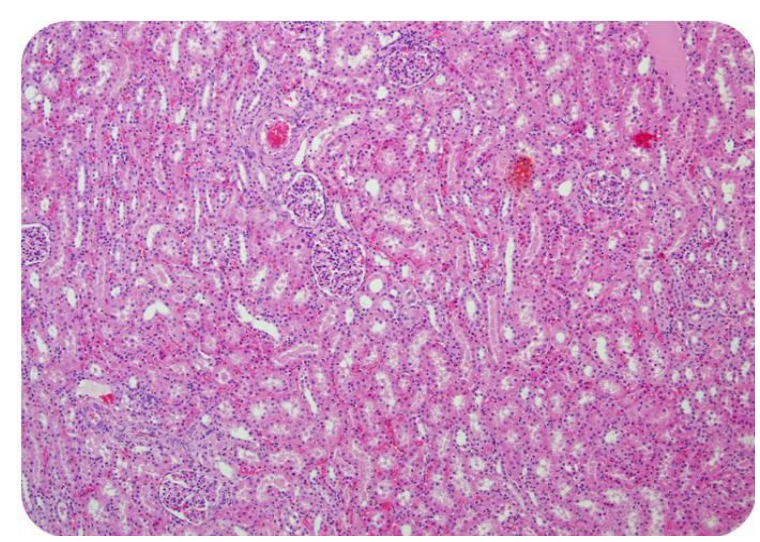

Figure 4: Bioptic sample IIIA (condition after transplantation in the group with the ROP).

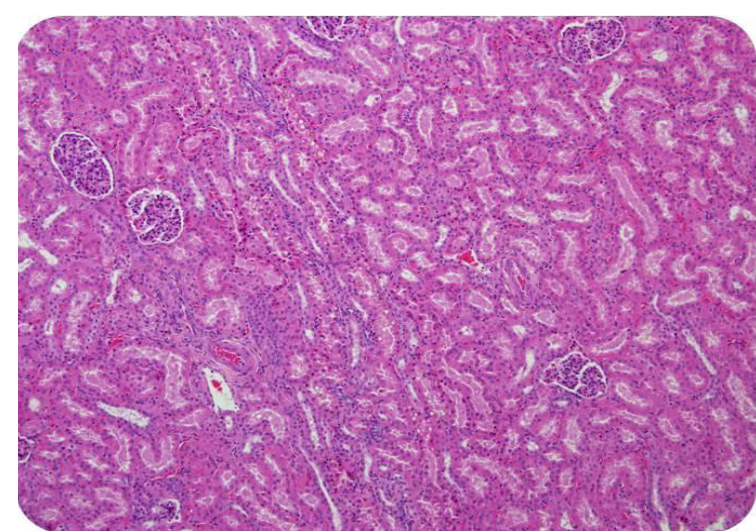

Figure 5: Bioptic sample IIIB (condition after transplantation in the group with intraarterial perfusion). 
Citation: Moláček J, Třeška V, Opatrný V, Matějka R, Baxa J (2015) Retrograde Oxygen Persufflation of Kidney: Experiment on an Animal. J Transplant Technol Res 5: 147. doi:10.4172/2161-0991.1000147

sweepers is also in question. In our opinion, the point is not to replace standard intraarterial perfusion by retrograde persufflation. Instead, a sort of combination of both methods seems to be promising. However, prior to clinical applications other experimental research is necessary.

\section{References}

1. Nishikido M, Noguchi M, Koga S, Kanetake H, Matsuya F, et al. (2004) Kidney transplantation from non-heart-beating donors: analysis of organ procurement and outcome. Transplant Proc 36:1888-1890.

2. Gomez-de-Antonio D, Varela A (2011) Non-heart-beating donation in Spain. Gen Thorac Cardiovasc Surg 59: 1-5.

3. Miranda-Utrera N, Medina-Polo J, Pamplona-Casamayor M, Passas-Martínez JB, Rodríguez-Antolín A, et al. (2015) Uncontrolled non-heartbeating donors (types $\mathrm{i}$-ii) with normothermic recirculation vs. heartbeating donors: Evaluation of functional results and survival. Actas Urol Esp pii: S0210-4806(15)00028-5.

4. Arnoux V, Dorez D, Muller M, Gignoux A, Valignat C, et al. (2014) Non-heartbeating renal donors: organization in a non-university hospitál Prog Urol 24 13-21.

5. Saito K, Takahara S, Nakagawa Y, Yagisawa T, Mieno MN, et al. (2013) Obstacles of non-heart-beating donor kidney transplantation in Japan to date and future perspectives. Transplant Proc 45: 2866-2870.

6. Geraci PM, Sepe V (2011) Non-heart-beating organ donation in Italy. Minerva Anestesiol 77: 613-623.

7. Gomez-de-Antonio D, Varela A (2011) Non-heart-beating donation in Spain. Gen Thorac Cardiovasc Surg 59: 1-5

8. Treska V, Hasman D, Reischig T, Certík B, Cechura M, et al. (2002) First kidney transplantation from a non-heart-beating donor in the Czech Republic. Ann Transplant 7: 45-49
9. Isselhard W, Witte J, Denecke $\mathrm{H}$, Berger M, Fischer JH, et al. (1974) Function and metabolism of canine kidneys after aerobic ischemia by retrograde persufflation with gaseous oxygen. Res Exp Med (Berl) 164: 35-44

10. Weber D, Tung LC (1986) Preservation of porcine livers by retrograde oxygen persufflation. Z Exp Chir Transplant Kunstliche Organe 19: 217-220.

11. Rolles K, Foreman J, Pegg DE (1984) Preservation of ischemically injured canine kidneys by retrograde oxygen persufflation. Transplantation 38: 102-106.

12. Rolles K, Foreman J, Pegg DE (1989) A pilot clinical study of retrograde oxygen persufflation in renal preservation. Transplantation 48: 339-342.

13. Isselhard W, Berger M, Denecke H, Witte J, Fischer JH, et al. (1972) Metabolism of caninekidneys in anaerobic ischemia and in aerobic ischemia by persufflation with gaseous oxygen.Pflugers Arch 337: 87-106.

14. Isselhard W, Denecke H, Stelter W, Berger M, Sachweh D, et al. (1973) Function andmetabolism of canine kidneys after aerobic ischemia by orthograde persufflation with gaseousoxygen. Res Exp Med (Berl) 159: 288-297.

15. Isselhard W, Witte J, Denecke H, Berger M, Fischer JH, et al. (1974) Function and metabolism of canine kidneys after aerobic ischemia by retrograde persufflation with gaseous oxygen. Res Exp Med (Berl) 164: 35-44.

16. Treckmann JW, Paul A, Saad S, Hoffmann J, Waldmann KH, et al. (2006) Primary organ function of warm ischaemically damaged porcine kidneys after retrograde oxygen persufflation. Nephrol Dial Transplant 21: 1803-1808.

17. Treckmann J, Nagelschmidt M, Minor T, Saner F, Saad S, et al. (2009) Function and quality of kidneys after cold storage, machine perfusion, or retrograde oxygen persufflation: results from a porcine autotransplantation model. Cryobiology 59: 19-23.

18. Suszynski TM, Rizzari MD, Scott WE, Tempelman LA, Taylor MJ, et al (2012) Persufflation (or Gaseous Oxygen Perfusion) as a Method of Organ Preservation. Cryobiology 64: 125-143. 